\title{
Advancement in Ocular Drug Delivery System to Overcome Ocular Barrier
}

\author{
Supriya Nikam*1, Abhilasha Ghule ${ }^{2}$, Akash Inde ${ }^{3}$, Anjali Jambhulkar ${ }^{4}$ \\ Sinhgad Institute of Pharmacy, Narhe, Pune, Maharashtra, India. \\ *Corresponding author's E-mail: sdeshmukh3105@gmail.com
}

Received: 04-10-2021; Revised: 26-11-2021; Accepted: 03-12-2021; Published on: 20-12-2021.

\begin{abstract}
The Ocular drug delivery system (ODDS) is the prominently challenging system faced by pharmaceutical researchers. Ophthalmic preparations are available in buffered, sterile and isotonic solutions. For the ocular delivery of drugs, various types of dosage forms are prepared and dispensed. As the drops are easier for the administration likewise more prescribed dosage form is the eye drop solution. For obtaining prolonged therapeutic effect ointment, suspensions and gelled systems are also used. The presence of various barriers as anatomical, physiological and physiochemical barriers makes difficulties in delivery of drugs in at the intended sites. Scientists invented alternate delivery routes to direct access at intended target sites. Second invention involves development of novel drug delivery systems providing better permeability, treatability and controlled release at target site. The liposomal delivery is beneficial because they have the ability of envelopment and both hydrophobic and hydrophilic drugs are suitable for delivery to both the anterior and posterior segment of the eye. Therefore, the uses of this alternative approach become quite a necessary. This formulation of novel devices will definitely help to the overcome ocular barriers and side effects with conventional topical drops. Current reviews on the conventional formulations of ocular delivery and their advancements followed by current nanotechnology based on the formulation developments. The recent incident with other ocular drug delivery planning consists of in situ gels, implants, contact lens and nano wafers are discussed. Drug delivery at ophthalmic route has been proven significant advancement for the future perspectives.
\end{abstract}

Keywords: Ocular, Nanocarriers, Liposomes, Barriers for ocular delivery.

QUICK RESPONSE CODE $\rightarrow$

DOI:

10.47583/ijpsrr.2021.v71i02.015

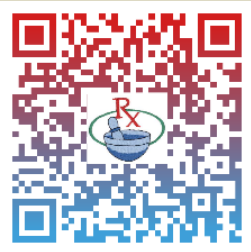

DOI link: http://dx.doi.org/10.47583/ijpsrr.2021.v71i02.015

\section{INTRODUCTION}

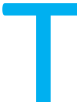

he eye is a complex organ with the unique anatomy and physiology. The structure of an eye is divided into two main parts: Anterior segment and posterior segment. Anterior segment of the eye occupies about onethird portion and the remaining portion is involved by the posterior segment of the eye. Tissues like cornea, conjunctiva, aqueous humor, iris, ciliary body and lens make up the anterior portion of the eye. ${ }^{1-6}$ Back of the eye or posterior segment of the eye includes sclera, choroid, retinal pigment epithelium, neural retina, optic nerve and vitreous humor. The anterior and posterior segment of eye is affected by various vision related diseases. ${ }^{7-9}$ For treatment of various eye ailments topical instillation are most widely preferred non-invasive route of drug administration, affecting. Conventional dosage forms like eye drops serves for $90 \%$ of the marketed ophthalmic formulation. ${ }^{10}$

The transscleral drug delivery with periocular administration route is evolved by an alternative mode of drug delivery to the posterior ocular eye tissues. Although transscleral delivery is easy, less invasive and patient compliant, drug permeation is adjusted by ocular static and dynamic barriers. Ocular barriers to transscleral drug delivery involves: static barriers i.e. sclera, choroid and retinal pigment epithelium (RPE), and dynamic barriers, i.e., lymphatic flow in the conjunctiva and episclera, and the blood flow in conjunctiva and choroid. 11,12

\section{THE ANATOMY AND PHYSIOLOGY OF AN EYE}

The human eye is essential sense organ of the body with quite complex anatomy. Eye is able to refract light and produces focus image they can stimulate nervous system and enables the ability to see.

\section{The structure and different parts of the Eye:}

Aqueous Humour: It is a jelly-like substance located in the anterior chamber of the eye.

Choroid: It is the layer behind the retina and absorbs unused radiation.

Ciliary Muscle: It is a ring-shaped muscle it is attached to the iris. The shape of the iris is controlled by the contraction and relaxation of ciliary muscle.

Cornea: It is a clear transparent epithelial membrane. The Light rays passes through the cornea to reach the retina. The cornea is convex and is involved in refracting light rays to focus them on the retina. 
Fovea: It is a small (approx. $1.5 \mathrm{~mm}$ in diameter) in the retina. It is the part of the retina in which high-resolution vision of fine detail can be done.

Hyaloid: The hyaloid diaphragm divides the aqueous humour from the vitreous humour.

Iris: Iris is the visible part of the eye and extends anteriorly from the ciliary body, lying behind cornea and in front of the lens. Iris divides the anterior segment of the eye into anterior and posterior chambers in that they contain aqueous fluid secreted by ciliary body. Parasympathetic and sympathetic nerves are supplied to iris. Parasympathetic stimulation constricts the pupil and sympathetic stimulation dilates the pupil. ${ }^{18}$

Lens: It is the lens of the eye which is a flexible unit which consists of various tissue layers enclosed in a tough capsule. It is suspended from the ciliary muscle through the zonule fibres.

Optic Nerve: Optic nerve is a second cranial nerve and it is responsible for the vision. Each nerve contains approx. 1 million fibres transmitting information from the Rod and Cone cells from the retina.

Papilla: It is also known as blind spot and is located at the position from which the optic nerve leaves the retina.

Pupil: It is the aperture through which light and hence the images we see and perceive enters the eye. This is formed by the iris.

Retina: It is described as the screen on which an image is formed by light which is passed into the eye via the cornea, aqueous humour, pupil, lens, hyaloids and finally the vitreous humour before reaching to the retina. The retina contains photosensitive elements (called rod and cones) that convert the light they detect into nerve impulses that are then sent onto the brain along the optic nerve. ${ }^{19}$

Sclera: it is the sclera is a tough white sheath around the outside of the eye-ball It consist of a membrane that maintains the shape of the eye and it provides the attachment to the extrinsic muscle of the eye.

Vitreous Humour: It is a jelly like substance (vitreous body).

\section{AN INSIDE LOOKS AT EYE:}

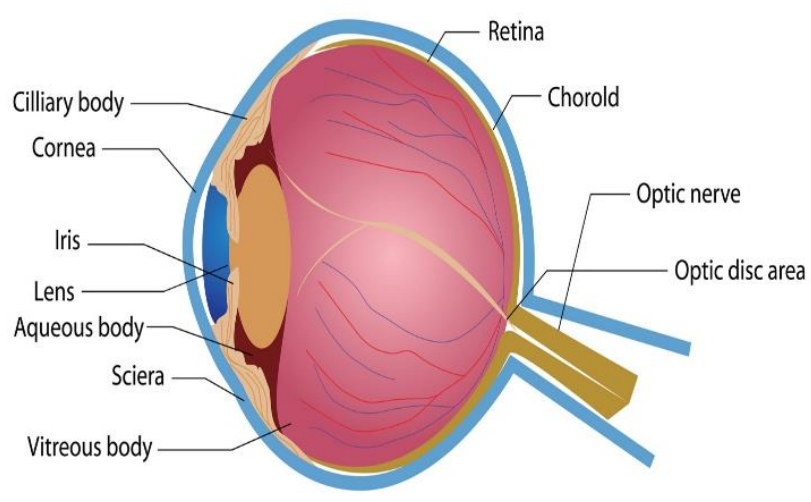

Figure 1: Anatomy of the Eye

\section{BARRIERS OF DRUG PERMEATION}

The reason behind the difficulty in acheiving relevant therapeutic doses within the eye is due presence of multiple barrier. Whenever the dosage form is administerd topically or systemically it faces multiple obstacle before reaching at the inteded site of action. Accordingly the result of bioavailability from topical route is usually only $1 \%-7 \%$ of the given dose. Such type of barriers are broadly classified into Anatomical barriers and Physiological Barriers.

\section{Anatomical Barrier}

Whenever the drug is topically administred it is either by corneal or via non corneal route. The cornea is a multilayered tissue composed of five sections: Epithelium, Bowman's membrane, Stroma, Descemet's membrane and Endothelium(principal barrier) which acts as a major barrier to hydrophilic drug transport. While the Stroma allows hydrophilic drugs to easily pass through but barrier for lipophilic drug. ${ }^{20}$ Thus, the drug should have optimum bioavailability with right balance between lipophilicity and hydrophilicity. Non corneal route bypasses the cornea by involving movement across sclera and conjunctiva (more permeable to hydrophilic drug).

\section{Cornea}

It is a largest static barrier which inhibits the transportation of drug molecule into the eye. Corneal epithelium selectively controls ocular drug penetration. As the lipophilic character of the epithelium, contributes as a barrier for $90 \%$ of the hydrophilic drug and for $10 \%$ of the lipophilic drug. As the cornea consists of epitheliumstroma-endothelium, that is equivalent to fat-water-fat structure, the penetration of non-polar compounds through the cornea depends on the oil-water partition coefficients of the drug molecules. The cornea act like a drug reservoir which slowly releases the drug into the aqueous humor. After corneal permeation, drugs are distributed from the aqueous humor to the intraocular tissues, i.e. iris ciliary body, lens, vitreous and choroids, retina and eliminates via aqueous humor turnover and venous blood flow in the anterior uvea. Ocular penetration via the sclera conjunctival route is more rapid (for a hydrophilic drug) than via the transcorneal route. ${ }^{22}$

\section{Conjunctiva}

It is a thin vascularized tissue, internally lining the eyelids and anterior sclera covering. It is composed of two layers: an outer epithelium that acts as the major penetration barrier and stroma. The outer apical epithelial cells of conjunctiva form tight junctions allowing paracelluar drug permeation across the cell layers. The conjunctival sclera stroma with blood, and lymph vessels are barrier to hydrophobic drugs. Intercellular spacing in the conjunctival epithelium is wider than corneal epithelium, thus the permeability of drugs across the conjunctiva is greater than across the cornea. However, drug absorption through the conjunctiva is still minimal due to the presence 
of conjunctival blood capillaries and lymphatics, which can cause significant drug loss into the systemic circulation by lowering the overall ocular bioavailability. ${ }^{23}$ The conjunctiva exhibits esterase activity expressing efflux proteins as glycoprotein on the cell membrane, involved in drug efflux from the cell cytoplasm.

\section{Physiological Barrier}

The primary line of defence of Eye is its tear film. The lacrimal fluid is an isotonic solution containing mixture of proteins (lysozyme) as well as lipids. If lacrimation is increased which leads to dilution of administered dose. Because of this drug concentration lowers which leads to diminished drug absorption. whichever results in rapid clearance from precorneal area by lacrimation and through nasolacrimal drainage which reduces the contact time between tissue and drug molecules, which automatically lowers the time for absorption leading to reduced bioavailability. Thus, the drugs which is administered as eye drop need to be isotonic and non irritating for prevention of precorneal loss. ${ }^{21}$

\section{Lacrimal Secretion}

This fluid maintains the conjunctiva and cornea moist and healthy by blinking action of eye and drained to nose, fluid is secreted by lacrimal glands. More than $75 \%$ of ophthalmic solution is lost via nasolacrimal drainage and absorbed systemically via conjunctiva, nasolacrimal drainage during blinking moves towards nasolacrimal duct which induces elimination of applied dosage form.

\section{Tear Film}

It is a three-layer structure composed of an external lipid layer, middle aqueous layer, and an inner mucin layer which covers the conjunctiva and the cornea. $\mathrm{pH} 7.4$ containing electrolytes, lipids, and proteins.

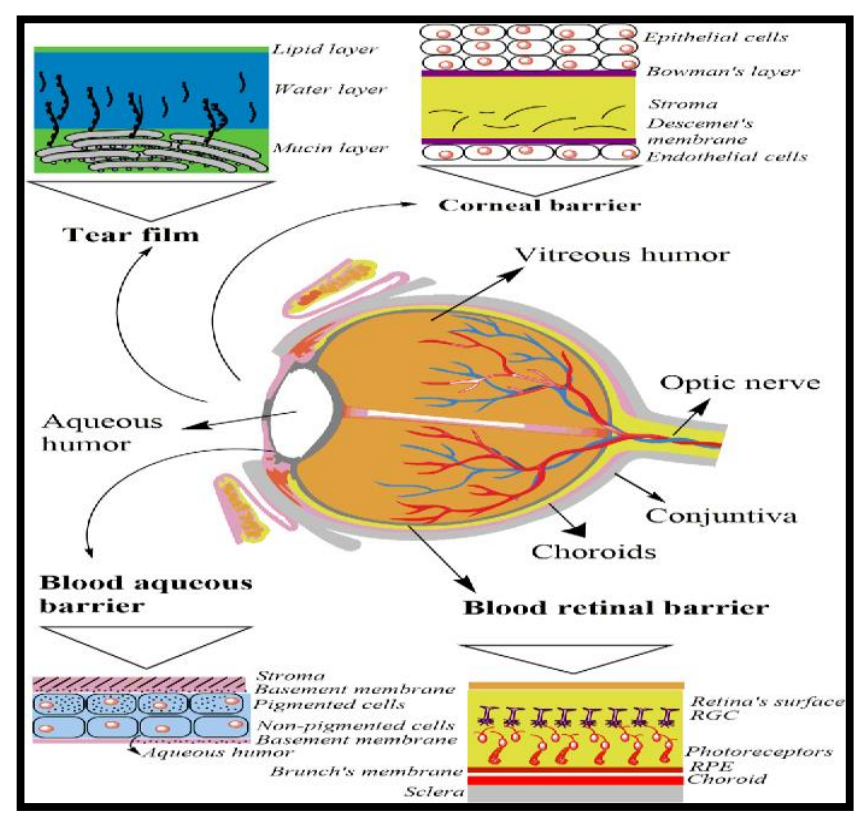

Figure 2: Anatomical and Physiological Barriers to ocular delivery system.
The instilled drug could bind proteins or be partially hydrolyzed by enzymes, reducing ocular bioavailability. Because of tear production (0.5-2.2 $\mu \mathrm{l} / \mathrm{min})$ and tear turnover which decreases contact time with ocular tissue by $1-2 \mathrm{~min}$. As a result only a slight percentage of the free drug applied on the corneal epithelium successfully penetrates through cornea. ${ }^{19}$

\section{Blood Aqueous Barrier}

The aqueous humor is protected by the blood aqueous barrier (BAB). It is formed by the iris and ciliary muscle vasculature endothelium and by the posterior iris and nonpigmented ciliary epithelium. This barrier is poorly permeable due to tight intracellular junctions, which limits the passage of substances with high molecular weight or high hydrophilicity. Ions and small solutes diffuse through space between cells. An active transporter also affects the passive diffusion of drug depending on their selectivity.

\section{Blood Retinal barrier}

Retina is a thin transparent tissue which forms the innermost layer of the eye and adheres to the choroid. The BRB hinders substance into the retina.

The inner BRB composed of retinal capillary endothelial cells which posses intercellular tight junctions and protects the retina from foreign particles. The outer BRB is composed of the retinal pigment epithelium(RPE), which is located between the photoreceptors and choriocapillaries. Lipophilic molecules can transfer efficiently between choroid and retina by limiting drug delivery in inward (blood to vitreous) and outward (vitreous to blood). Hydrophilic compounds permeate through tight juctions (paracellular routes), while lipophilic drug cross the RPE via trancellular route. ${ }^{20}$

\section{Bruch's-choroid complex (BC complex)}

Choroid is a highly vascularised and innervated tissue supplying blood to retina. It is composed of a network of fenestrated capillaries and supported by Bruch's membrane, it is thin $(2-4 \mu \mathrm{m})$. BC complex poses a more critical barrier to drug delivery by the transscleral route than the sclera. The molecular size affects BC complex permeability.

\section{METHODS TO OVERCOME OCULAR BARRIER}

\section{1) Conventional ophthalmic dosage forms}

Solutions are widely used as dosage forms for topical delivery of drug to the eye. These factors should be considered in formulating ophthalmic solutions are solubility, ocular toxicity, and effect of $\mathrm{pH}$, tonicity, buffer capacity, viscosity and compatibility with other ingredients in the formulation.

\section{(i) Viscosity enhancers}

Polymers are added in ophthalmic drug solutions which increase the viscosity and relates to a slower elimination from the preocular area that leads to improve precorneal residence time and more transcorneal penetration of the 
drug into the anterior chamber. The polymers used are methylcellulose, polyvinyl alcohol, polyvinylpyrrolidone (PVP), hydroxyethylcellulose, hydroxypropyl methylcellulose (HPMC), and hydroxypropylcellulose. Natural polymers like veegum, alginates, xanthan gum, gelatin, acacia, and tragacanth are also used. ${ }^{22}$

\section{(ii) Eye ointments}

Ointments are formulated by using mixtures of semisolid and solid hydrocarbons (paraffin) that have a melting or softening point close to body temperature and they are non-irritating to the eye. Ointments are simple bases, where the ointment forms one continuous phase, or compound bases where a two-phased system (emulsion) is employed. After instillation in the eye, ointments break up into small droplets and remains as a depot of drug in the cul-de-sac for long time. Ointments have poor patient compliance because blurring of vision and occasional irritation. ${ }^{24}$

\section{(iii) Penetration enhancers}

To increase the permeability of the corneal epithelial membrane the transport characteristics of the cornea can be maximized, to improve ophthalmic drug bioavailability, this approach is used which increases the permeability of the cornea called penetration enhancers or absorption promoters. The transport process from the cornea to the receptor site is a rate-limiting step, and permeation enhancers increase corneal uptake. ${ }^{25}$

\section{(iv) Prodrug}

The prodrug is used to enhance corneal drug permeability through modification of the hydrophilicity (or lipophilicity) of the drug. Within the cornea the prodrug is either chemically or enzymatically metabolized to the active parent compound. The ideal prodrug should not only have to increased lipophilicity and a high partition coefficient, but it must also have high enzyme susceptibility.

Example: An antiviral medication as acyclovir is suitable prodrug.

\section{(v) Ocular Iontophoresis}

It is a method for active delivery of drug using mild electric charges for effective delivery through the ocular barriers. lontophoresis enhances ocular drug delivery by using electroporation (electric field-induced ocular tissue structure alteration and pore formation), electrophoresis (direct application of electric field), and electro-osmosis (convective solvent flow through an applied electric potential). lontophoresis is a noninvasive method it is advantageous over invasive techniques required surgical interventions. This technique of drug permeation is used for anterior and posterior ocular disorders by using transcorneal and trans-scleral routes. Transcorneal iontophoresis is used for treatment of anterior segment disorders such as corneal ulcers, dry eye disease, ocular inflammation, keratitis, and ocular uveitis. Transcorneal iontophoresis is not suitable for posterior segment delivery due to the presence of barriers such as the lens diaphragm and iris-ciliary.

\section{NANOCARRIERS IN OCULAR DRUG DELIVERY SYSTEM}

Nanocarriers are the distinct particulate systems of particle size (10-1000 nm) with a specific surface charge. Various nanocarriers are explored for the use in ocular applications. The surface charge of nanocarriers contributes to their conjugation and retention at the specific site. The surface charge is measured as zeta potential. Zeta potential is defined as the potential difference between the surface charge of a nanocarrier and opposite charge is derived from the medium that is arranged around the particle. This is responsible for the stability of nanodispersion. ${ }^{26}$ If two particles are having high zeta potential of same charge, they will repel each other. In case of ophthalmic delivery, the cornea and conjunctiva have a negative charge on the surface so, the cationic nanoparticles are attracted towards electrostatic interactions. This leads to retention of the cationic nanoparticles on negatively charged ocular tissues to achieve topical drug delivery to the anterior eye region.

\section{1) Liposomes}

Liposomes are the lipid vesicles with one or more phospholipid bilayer that entrap water in their interior, thus amphiphiles self-assemble in aqueous layers to form single or multiple concentric bilayers. The size of liposomes usually ranges from 0.08 to $10 \mu \mathrm{m}$ and based on the size and phospholipid bilayers, liposomes can be classified as small unilamellar vesicle $(10-100 \mathrm{~nm})$, large unilamellar vesicles (100-300nm) and multilamellar vesicles (contains more than one bilayer). ${ }^{15}$ For ophthalmic applications, liposome represents ideal delivery systems due to excellent biocompatibility, cell membrane like structure and ability to encapsulate both the hydrophilic and hydrophobic drugs. Liposome demonstrated good effectiveness for both anterior and posterior segment ocular drug delivery in several research studies. ${ }^{14}$

\section{2) Hydrogels}

Hydrogels are the network of cross-linked polymer chains which are biocompatible in the intraocular space. ${ }^{16}$ Chemical processes occur because of the covalent bonds between polymer chains, where the physical processes occur because of ionic, hydrogen, hydrophobic, and van der Waals forces. Because these bonds gives stability to sustained release of a drug and maintain the drug concentration. For natural polymers, polysaccharides, such as hyaluronic acid, chitosan, and dextran, are widely used to design hydrogels. Hydrogels are negatively charged, and protein degradation and drug release in the vitreous chamber can be controlled by modifying the physicochemical properties of the hydrogel molecule. Chitosan is a cationic nanocarrier it interact with anionic polymers to form hydrogels. These synthetic polymers, PEG, PVA, PAM, and PLGA are commonly used to produce hydrogels. ${ }^{17}$ 


\section{3) Dendrimers}

A dendrimer is the class of macromolecule with the highly branched three-dimensional structure having structural uniformity, high compatibility, multi-valency and hence display an important role in emerging field of nanomedicine. To avoid obstacle related to solubility and bioavailability, dendrimer acts as unimolecular micelle capable of encapsulating and solubilizing drug into the void spaces of the interior 35 . This highly branched structure of dendrimers allows incorporation of wide range of drugs, hydrophobic as well as hydrophilic. In ocular drug delivery dendrimer provide hopeful results were reported.

\section{4) Nanoparticle}

Nanoparticles are the colloidal carriers with particle size 10-1000nm. Nanoparticle generally composed of lipids, proteins, natural or synthetics polymers such as albumin, sodium alginate, chitosan, poly (lactide-co-glycolide) (PLGA), polylactic acid (PLA) and polycaprolactone31. Nanoparticle have gained attention for ocular drug delivery and research scientist have tried to develop drug loaded nanoparticles for the delivery both posterior and anterior segment. ${ }^{7}$

\section{5) Nanomicelles}

Nanomicelles are the most widely used carrier systems for formulating therapeutic agents in to clear aqueous solutions. These nanomicelles are made up of amphiphilic molecules. These molecules are surfactant or polymeric in nature. Currently, tremendous interest is being showed towards development of nanomicellar formulation based technology for ocular drug delivery. Micellar formulation increases the bioavailability of the therapeutic drugs in ocular tissues. Nanomicelles retained in systemic circulation for longer time and accumulate at the diseased tissue. ${ }^{4}$

\section{6) Contact Lens}

Contact lenses have thin, and curved shaped plastic disks which are designed to cover the cornea. After application of contact lens they adhere to the tear film over the cornea due to the surface tension.

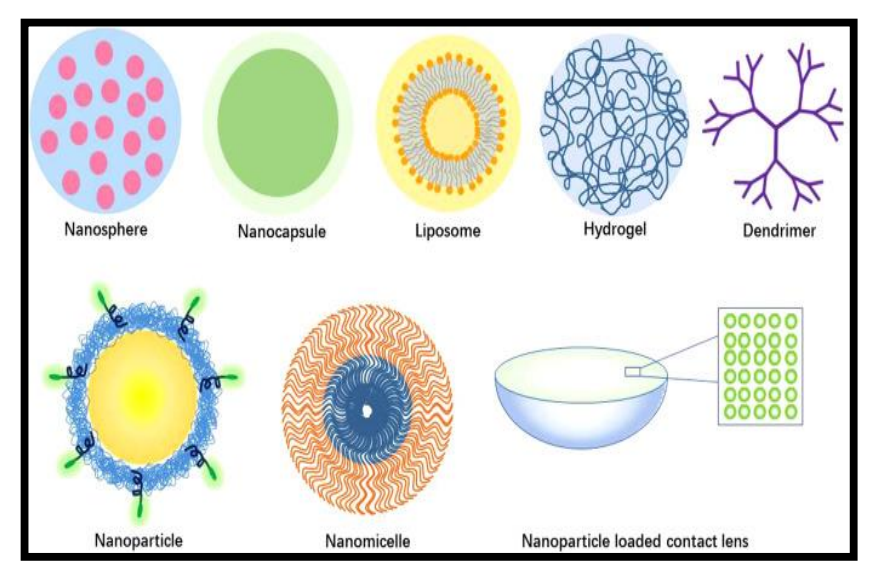

Figure 3: Various Nanocarriers used in Novel Drug Delivery System.
Drug loaded on contact lens for ocular delivery of numerous drugs such as $\beta$-blockers, antihistamines and antimicrobials. In presence of contact lens, drug molecules have longer residence time in the post-lens tear film which ultimately leads to higher drug flux through cornea with less drug inflow into the nasolacrimal duct. ${ }^{2}$ Drug is loaded by soaking contact lens into drug solutions. These soaked contact lenses have higher efficiency in delivering drug compared to conventional eye drops. ${ }^{3}$

\section{7) Niosomes}

Niosome is a bi-layered drug carrier system formed by selfassociation of non-ionic surfactant and cholesterol in aqueous phase which can entrap both hydrophilic and lipophilic drug. Niosomes in topical ocular delivery are preferred over the other vesicular systems because they are chemically stable, have low toxicity because of their nonionic nature, handling of surfactants does not require special precautions and condition, exhibit's special structural characteristics. Niosomes are also used in modified form i.e., Discosomes (12-16 $\mu \mathrm{m})$ in ophthalmology. Discosomes contains non-ionic surfactant (solulan C24). These vesicles fit better in the cul-de sac of the eye and are not drained into systemic circulation because of their large size. $^{8}$

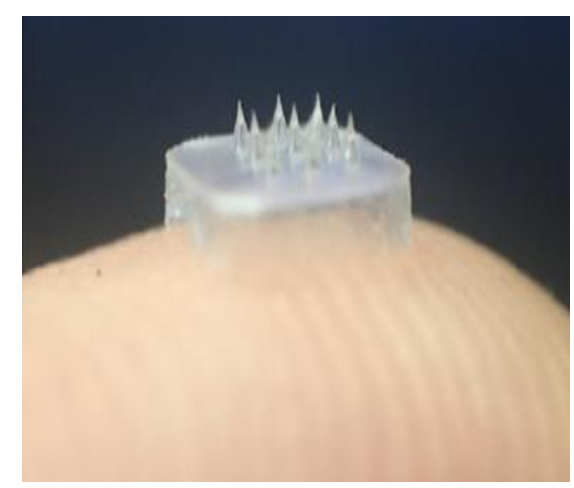

Figure 4: Microneedle

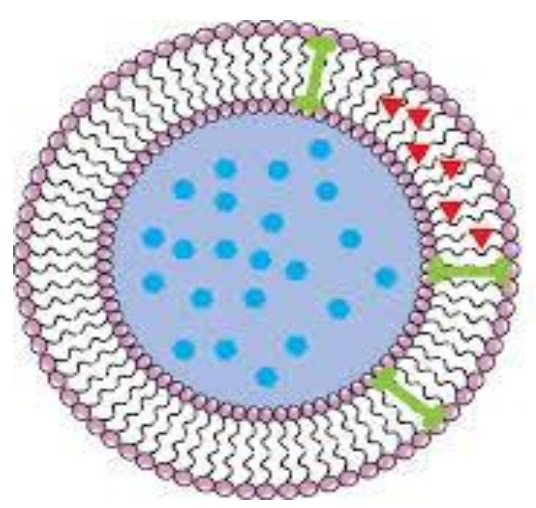

Figure 5: Niosome

\section{8) Microneedles}

Microneedles are the invention for microvascular injection and method for treating glaucoma using the device. It consists of blunted needle connected to a flexible tapered tubing, at end of which is positioned a microneedle or micropipette for inserting into small blood vessels. This is 
a minimally invasive technique applied for the ocular drug delivery of hydrophilic and hydrophobic drugs. Microneedles may deliver drugs into the sclera to treat diseases in the posterior segment that avoids the complications associated with intraocular injection and systemic administration. ${ }^{13}$

\section{9) Nano wafers}

Nano wafers are the small transparent rectangular membrane or circular discs in which drug loaded into nanoreservoirs which can be smeared to the ocular surface using a fingertip. Controlled release from nano-wafers can be improve, can increase the residence time and contact time of the drug with corneal and conjunctival tissues. ${ }^{7}$ It also improves the drug bioavailability and heals the injured the destroyed corneal surface mainly seen in CNV and dry eye disease.

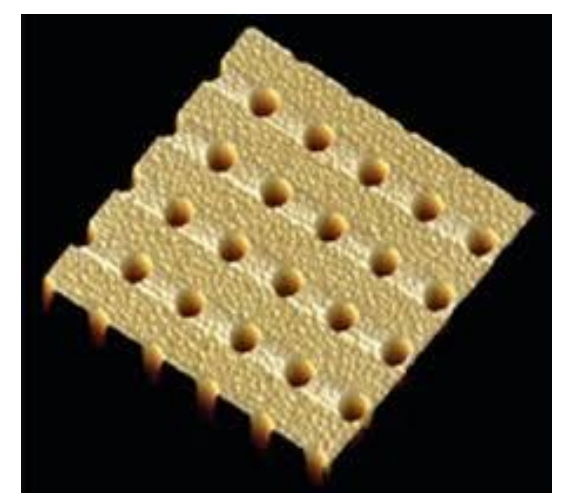

Figure 6: Nanowafer

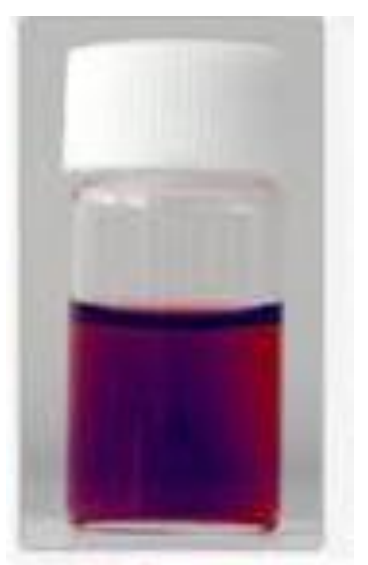

Figure 7: Nanosuspension

\section{0) Nanosuspensions}

Nanosuspensions are colloidal dispersion of submicron drug particles stabilized by polymer or surfactant. They deliver the hydrophobic drugs. For ocular delivery, there are many advantages such as sterilization, ease of eye drop formulation, less irritation, increase precorneal residence time and enhancement in ocular bioavailability of drugs which are insoluble in tear fluid. The efficacy of nanosuspensions to improve ocular bioavailability of glucocorticoids has been demonstrated in several research studies. Glucocorticoids such as prednisolone, dexamethasone and hydrocortisone are recommended in the treatment of inflammatory conditions affecting anterior segment ocular tissues. ${ }^{1}$

\section{1) In situ Gelling system}

Ophthalmic in-situ gelling system consists of environmentally sentient polymer that will be changed structurally with the small alteration in specific condition like $\mathrm{pH}$ change, Temperature modification, solvents exchange and ionic strength in the environment. In situ forming gel are liquid at time of installation into eyes and then underwent rapid gelation in the cul-de-sac of the eye to form viscoelastic gel in response to environmental changes. Hence, the residence time of the formed gel will be extended and release the drug in sustained manner which helps to improve bioavailability, minimized systemic absorption, and reduced dose regimen resulting to patient compliance. Even, in situ gelling system provides others potential advantages like simple manufacturing process, ease to administer; releasement of accurate dose can obtain by in situ gelling system.

\section{2) Microemulsion}

Microemulsions are novel ocular delivery systems which are dispersions of water and oil along with a surfactant. Microemulsions have advantages of higher thermodynamic stability, improved solubility, and improved corneal permeation. The parameters that affect the stability of the microemulsion system are selection of aqueous phase, organic phase, and surfactant/ cosurfactant systems. ${ }^{6}$ Tears can be attractive in ocular delivery systems as they provide the fluidity with increased viscosity after application, which increases ocular retention while retaining therapeutic efficiency. Drugs used in ophthalmic delivery are timolol, sirolimus, and chloramphenicol was formulated in various microemulsions with improved stability, solubility, and bioavailability. ${ }^{5}$

\section{3) lontophoresis}

lontophoresis involves application for a low-density electrical current to increase drug transport across skin, nail, and eye structures. Iontophoresis for ocular delivery was first performed by Wirtz, in 1908 he studied topical ocular delivery of zinc salts for the treatment of corneal ulcers. It is a noninvasive method for transferring of ionized drugs through membranes with low electrical current. Two mechanisms are involved for movement of drugs across the membrane: migration and electroosmosis. Classification of Ocular Iontophoresis: as transcorneal, corneoscleral, or trans-scleral iontophoresis. Some drawbacks are it requires repeated administrations, no sustained half-life and side effects i.e., mild pain in some cases. Merits of iontophoresis are fast, painless and safe system, and to a specific site delivery of high concentrations of drug. ${ }^{5-6}$ 


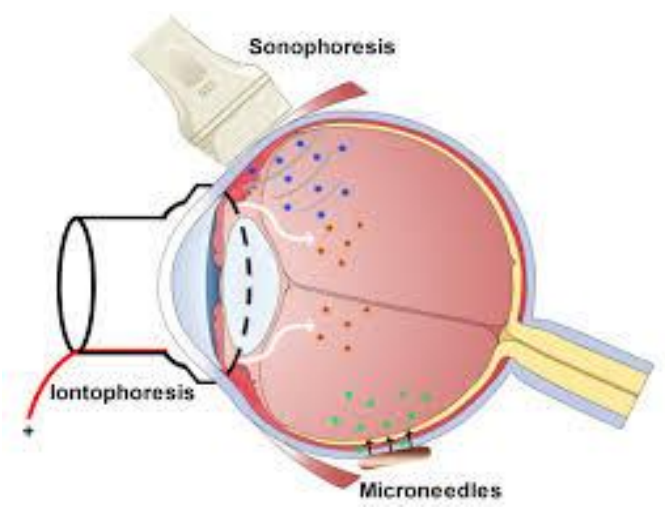

Figure 8: Ocular lontophoresis

\section{4) Sonophoresis}

Sonophoresis or ultrasound, involves application of ultrasound of frequencies higher than $20 \mathrm{khz}$ to increase transdermal and ocular permeation. An ultrasound is applied over the epithelium through a coupling medium like emulsion or suspension of any formulation which allows the propagation of acoustic field. Sonophoresis enhances intra scleral delivery of fluorescein isothiocyanate conjugated to serum albumin in an ex- vivo rabbit eye model, by using the frequency $1 \mathrm{MHz}$ with an intensity of $0.05 \mathrm{~W} / \mathrm{cm} 2$ and 30 s exposure time. Results of above experiment shows that sonophoresis increased the transcleral permeation of the protein without damaging ocular tissues. ${ }^{27}$

\section{5) Phototherapy}

It involves 'light or radiant energy-induced treatment.' Depends on the wavelength, intensity of light, and duration of exposure, tissues can either absorb the energy (photocoagulation, thermotherapy, and PDT) or undergo ionization. Photocoagulation is the thermal denaturation of tissues. A tumour which contains haemoglobin and melanin can absorb argon or krypton laser wavelengths. Thermotherapy, the tumour is heated up to a temperature of $60-65^{\circ} \mathrm{C}$ by an infrared diode laser applied via the pupil. Thus, rise in temperature causes cytotoxicity. Photodynamic therapy PDT it is a non-thermal, photobiochemical process which involves in site-specific vascular occlusion and tumour destruction with minimal damage to adjacent structures. ${ }^{28}$

\section{CONCLUSION}

Drug delivery to the targeted ocular tissues faced major challenge to ocular scientist, for decades. Administration of drug solutions as topical drop with conventional formulations was associated with many drawbacks which started the introduction about different carrier systems for ocular delivery. In the therapy of eye disease the ocular insert represents a successful advancement. This ocuserts provides many advantages to improve patient compliance by reducing the frequency of dosing, by providing sustained and controlled drug delivery and reduces the dosing frequency and thereby reduces the adverse effects of the drug. Consequently, it seems logical to consider that nonconventional approaches such as nanotechnology, microspheres, liposomes, in situ forming gel, and iontophoresis for effective delivery and to then enhance ocular absorption and reduce side effects. So, there will be an urgent need for the development of novel noninvasive drug-delivery systems that can manage to overcome ocular barriers, sustain drug release, and maintain effective drug levels at the back of the eye.

\section{REFERENCES}

1. Patravale VB, Date AA, Kulkarni RM. Nanosuspensions: a promising drug delivery strategy, Journal of Pharmacy and Pharmacology, 2004;56: 827-840. [PubMed: 15233860]

2. Gupta H, Aqil M. Contact lenses in ocular therapeutics, Drug Discovery Today, 2012; 17:522-527. [PubMed: 22305935]

3. Gulsen D, Li CC, Chauhan A. Dispersion of DMPC liposomes in contact lenses for ophthalmic drug delivery, Current Eye Research, 2005; 30:1071-1080. [PubMed: 16354620]

4. Cholkar K, Patel A, Vadlapudi DA, Mitra AK. Novel Nanomicellar Formulation Approaches for Anterior and Posterior Segment Ocular Drug Delivery, Recent Patents on Nanomedicine. 2012; 2:82-95. [PubMed: 25400717]

5. Li CC, Abrahamson M, Kapoor $Y$ and Chauhan A. Timolol transport from microemulsions trapped in HEMA gels. Journal of Colloidal Interface and Sciences, 2018; 315: 297306.

6. Kaur IP and Kanwar M. Ocular preparations: The formulation approach, Drug Development and Industrial Pharmaceutics, 2018; 28: 473-93.

7. Bian F, Shin CS, Wang C, Pflugfelder SC, Acharya G, and De Paiva CS. Dexamethasone drug eluting nanowafers control inflammation in alkali-burned corneas associated with dry eye, Investigative Ophthalmology Visual Science, 2018; 57:3222-3230.

8. Watrad K, Mitra A. Drug Delivery in Ocular Disease: Barrier and Strategies. World Journal of Pharmacology, 2013; 2:7883.

9. Bourlais $C L$, Acar L, Zia H, Sado PA, Needham T, Leverge R. Ophthalmic drug delivery systems-recent advances. Progress in Retinal Eye Research, 1998; 17:33-58. [PubMed: 9537794]

10. Gulsen D, Chauhan A. Ophthalmic drug delivery through contact lenses, Investigative Ophthalmology Visual Science, 2004; 45:2342-2347. [PubMed: 15223815]

11. Kim SH, Lutz RJ, Wang NS, Robinson MR. Transport barriers in transscleral drug delivery for retinal diseases, Ophthalmic Research. 2007; 39:244-254, [PubMed: 17851264]

12. Lee SJ, Robinson SB, Robinson MR, Csaky KG, Kim H. Evaluation of clearance mechanisms with transscleral drug delivery. Investigative Ophthalmology Visual Science. 2010; 51:5205-5212, [PubMed: 20484583]

13. Jiang J, Gill HS, Ghate D, McCarey BE, Patel SR, Edelhauser $H F$, Prausnitz MR. Coated microneedles for drug delivery to the eye. Investugative Ophthalmology Visual Science. 2007; 48:4038-4043.10.1167/iovs.07-0066 [PubMed: 17724185] 
14. Patel A, Cholkar K, Agrahari V. Ocular drug delivery systems: an overview, World Journal of Pharmacology 2013; 2(2):4764.

15. Kaur IP, Garg A, Singla AK. Vesicular systems in ocular drug delivery: an overview. International Journal of Pharmacology. 2004; 269: 1-14.

16. Burdick JA, Prestwich GD. Hyaluronic acid hydrogels for biomedical applications. Advanced Materials. 2011; 23: 4156.

17. Kirchhof S, Goepferich AM, Brandl FP. Hydrogels in ophthalmic applications. European Journal of Pharmaceutics and Biopharmaceutics. 2015; 95: 227-238.

18. Born AJ, Tripathi B, Wolff's Anatomy of the eye and Orbit, $8^{\text {th }}$ edition. 211-232, 308-334. Chapman and Hall Medical, London.

19. Snell R, Lemp M., Clinical Anatomy of the Eye, 1989; 169175, Blackwell, Boston.

20. Huang AJ, Tseng SC, Kenyon KR. Paracellular permeability of corneal and conjunctival epithelia, Investigative Ophthalmology Visual Science. 1989; 30: 684-689. [PMID: 2703309]

21. Hughes PM, Mitra AK. Overview of ocular drug delivery and iatrogenic ocular cytopathologies, in Ophthalmic Drug Delivery Systems. A.K. Mitra, Editor. New York: Marcel Dekker, Inc, 1993: 1-27

22. Sasaki H, Igarashi $Y$, Nagano T, Yamamura K, Nishida K, Nakamura J. Improvement of the ocular bioavailability of timolol by sorbic acid, Journal of Pharmacy and Pharmacology. 2013; 47: 17-21.

23. Lee VH. Evaluation of ocular anti-inflammatory activity of Buteafrondosa, Journal of Pharmacy and Pharmacology, 2011; 11: 79-90.

24. Tirucherai GS, Dias C, Mitra AK. Effect of hydroxypropyl beta cyclodextrin complexation on aqueous solubility, stability, and corneal permeation of acyl ester prodrugs of ganciclovir. Journal of Ocular Pharmacology and Therapeutics. 2012; 18: 535-48.

25. Bhattacharjee A, Das JP, Adhikari P, Marbaniang D, Pal1P, Subhabrata Ray. Novel drug delivery systems for ocular therapy: With special reference to liposomal ocular delivery, European Journal of Ophthalmology. 2019; 29(1):113-126.

26. Gorantla S, Vamshi K, Rapalli A, Waghule T, Singh P, Dubey $S$. Nanocarriers for ocular drug delivery: current status and translational opportunity, The Royal Society of Chemistry. 2020; 1 : 27835-27855.

27. Buech G, Bertelmann E, Pleyer U, Siebenbrodt I, Borchert $\mathrm{HH}$. Formulation of sirolimus eye drops and corneal permeation studies, Journal of Ocular Pharmacology and Therapeutics. 2017; 23: 292-303.

28. Schmidt-Erfurth U, Hasan T, Gragoudas E, Michaud N, Flotte TJ, Birngruber R. Vascular targeting in photodynamic occlusion of subretinal vessels. Ophthalmology. 1995;1:1953-1961. [PubMed] [Google Scholar]

Source of Support: The author(s) received no financial support for the research, authorship, and/or publication of this article.

Conflict of Interest: The author(s) declared no potential conflicts of interest with respect to the research, authorship, and/or publication of this article.

For any question relates to this article, please reach us at: editor@globalresearchonline.net New manuscripts for publication can be submitted at: submit@globalresearchonline.net and submit_ijpsrr@rediffmail.com

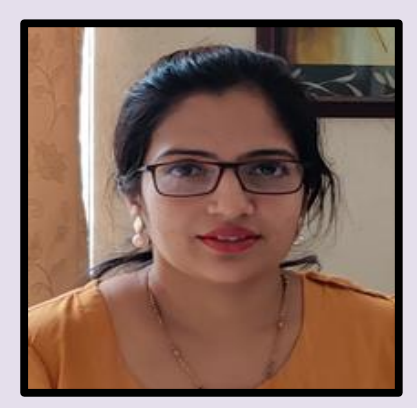

\section{Corresponding author biography: Mrs. Supriya Nikam}

Mrs. Supriya Nikam graduated at Mumbai University, Mumbai, Maharashtra, India and post-graduation from Savitribai Phule Pune University, Pune, Maharashtra, India. At postgraduation level did specialization in Pharmaceutics. She handles multiple NDDS projects, also guiding under graduate \& post graduate pharmacy students. 